(C) 2017 IEEE. Personal use of this material is permitted. Permission from IEEE must be obtained for all other uses, in any current or future media, including reprinting/republishing this material for advertising or promotional purposes, creating new collective works, for resale or redistribution to servers or lists, or reuse of any copyrighted component of this work in other works. 


\title{
Analytical Modeling of Manufacturing Imperfections in Double Rotor Axial Flux PM Machines: Effects on Back EMF
}

\author{
Baocheng Guo ${ }^{1}$, Yunkai Huang ${ }^{1}$, Youguang $\mathrm{Guo}^{2}$, Jianguo Zhu ${ }^{2}$ \\ ${ }^{1}$ Engineering Research Center for Motion Control of Ministry of Education, Southeast University, Nanjing 210096, China \\ ${ }^{2}$ School of Electrical, Mechanical and Mechatronic Systems, University of Technology Sydney, Sydney, Australian \\ Guobaocheng1986@gmail.com
}

\begin{abstract}
In order to detect the manufacturing imperfections of double rotor axial flux permanent magnet (DRAFPM) machine, the back EMF is calculated and compared via a fast and accuracy analytical model in this paper. At first, the radial and tangential flux density in the air gap under healthy condition is developed via Maxwell's equations and improved conformal mapping. After that, a bilinear mapping is introduced to calculate the flux density caused by manufacturing imperfections, which are classified as five cases. The back EMF at each case is calculated and compared with the healthy condition. At each section, FEM models are built to validate the analytical model, and the results showed that the analytical model predictions agree well with the observations from the FE results. Finally, the analytical model is verified via experimental results.
\end{abstract}

Index Terms - double rotor axial flux PM machine, Analytical model, Back EMF.

\section{INTRODUCTION}

Axial flux PM (AFPM) machines have a number of distinct advantages outstripping to the radial flux PM machines and widely used in the applications such as electrical vehicles (EV) and hybrid electric vehicles (HEV) where have limited space [1]. However, the AFPM becomes more difficult to design a rotor shaft mechanical joint with high mechanical integrity owing to the smaller contact surface between rotor and shaft, thus, the manufacturing imperfections are highly to occur because of improper design and assemble. Several approaches could be adopted to detect these imperfections. In this paper, the effects on the back EMF are chosen as research subject to investigate its influence. More detail can be seen in the full paper.

\section{FluX Density CALCUlation AND ANALYSIS}

A Quasi-3D method is adopted to convert 3D to 2D model in order to calculate the air-gap flux density, which is shown in Fig.1. The machine is divided into certain number of layers in order to consider the 3D structure.

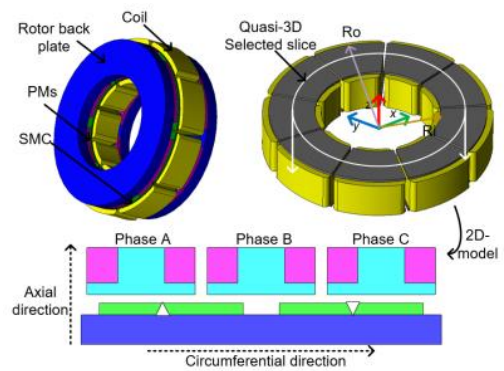

Fig.1 Construction of investigated AFPM (up) and the principle of quasi - 3D method (down).

Next, the Maxwell's equations are solved, the tangential and radial flux densities of air gap under slotless structure are obtained. After that, an improved conformal mapping, Schwarz Christoffel mapping is used to calculate the well-known Cater's factor. This numerical solution could relax some assumptions and consider the interaction between two adjust slots. The radial and tangential flux density at no-load and on load conditions are shown in Fig.2. More detail information could be seen in the full paper.
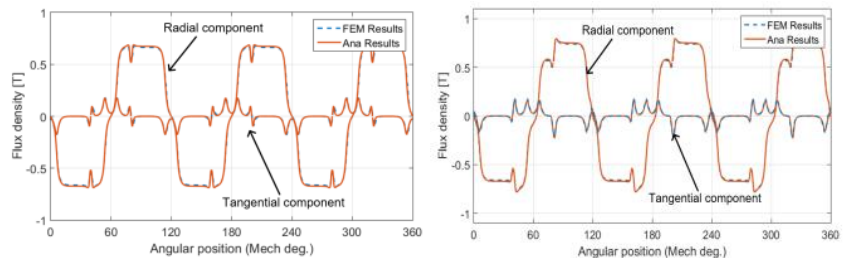

Fig.2. Radial and tangential components of flux density at the center of air gap of the PM at no-load (left) and rated load (right).

\section{BACK EMF CALCULATION AND ANALYSIS}

To evaluate the manufacturing imperfections, one of the five cases, the static eccentricity (SE), is selected in this digest. Fig.3 shows its topology and basic information.
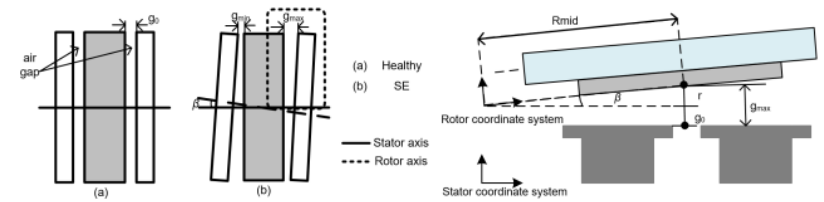

Fig. 3 The SE in AFPMs

The bilinear mapping is used to mapping the flux density to the eccentricity condition. The flux density and the back EMF of SE is shown in Fig.4. The back EMFs of the other four cases could be seen in full paper.
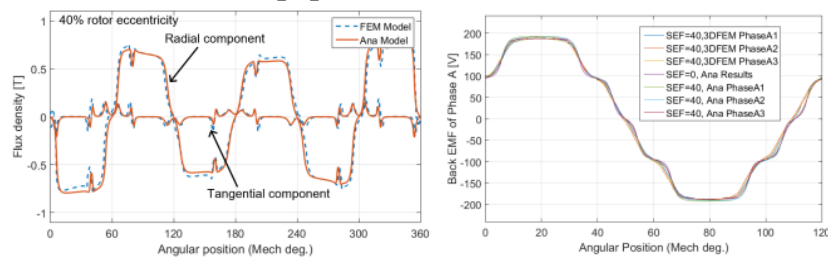

Fig.4. Components of flux density under SE (left) and its back EMF (right).

\section{CONCLUSION}

In this paper, a complete analytical model is presented to investigate the manufacturing imperfections, the back EMF under SE, un-uniform air gap, etc., are calculate in order to detect the improper assembles during manufacture.

\section{REFERENCES}

[1] F. Giulii Capponi, G. De Donato, and F. Caricchi, "Recent Advances in Axial-Flux Permanent-Magnet Machine Technology," IEEE Trans. Ind. Appl., vol. 48, no. 6, pp. 2190-2205, Nov. 2012. 